\title{
REVIEW
}

\section{Relationship between obsessive compulsive disorder and cortisol: Systematic review and meta-analysis}

\author{
João Sousa-Limaa,b,1, Pedro Silva Moreiraa,b,1, \\ Catarina Raposo-Lima ${ }^{a, b}$, Nuno Sousa ${ }^{a, b}$, Pedro Morgado ${ }^{a, b, *}$
}

${ }^{a}$ Life and Health Sciences Research Institute (ICVS), School of Medicine, University of Minho, Campus de Gualtar, 4710-057 Braga, Portugal

bICVS-3Bs PT Government Associate Laboratory, Braga/Guimarães, Portugal

Received 4 October 2018; received in revised form 27 July 2019; accepted 3 September 2019

\section{KEYWORDS \\ OCD; \\ Cortisol; \\ Glucocorticoids; \\ Stress; \\ Hypothalamic- \\ pituitary-adrenal \\ axis}

\begin{abstract}
Altered stress response and consequent elevated levels of circulating glucocorticoids have been found in neuropsychiatric disorders such as depression or anxiety disorders and proposed to also play a role in the pathophysiology of obsessive-compulsive disorder (OCD). Despite the observation that stressful events may precede the disease onset or even exacerbate its symptoms, studies in this field do not always report consistent results regarding the cortisol profile of OCD patients. As such, a systematic review and meta-analysis was developed to clarify this issue. This systematic review and meta-analysis was elaborated according to the PRISMA method. The analytical procedures were implemented using Metafor package in R software. Nineteen studies were included in the systematic review and 18 were included in the meta-analysis. The meta-analytic results demonstrated that OCD patients had significantly higher cortisol levels compared to controls $(d=0.76, \mathrm{SE}=0.146, p<0.001)$. For studies using the average of multiple assessments, the standardized coefficient was significantly higher when compared to studies focusing on single measurements. Both the systematic review and meta-analysis suggest that cortisol levels are significantly higher in OCD patients than healthy individuals.
\end{abstract}

(C) 2019 Elsevier B.V. and ECNP. All rights reserved.

\footnotetext{
* Corresponding author at: Life and Health Sciences Research Institute (ICVS), School of Medicine, University of Minho, Campus de Gualtar, 4710-057 Braga, Portugal.

E-mail address: pedromorgado@med.uminho.pt (P. Morgado).

${ }^{1}$ These authors contributed equally.
}

\section{Introduction}

Obsessive-compulsive disorder (OCD) is a common and debilitating mental disorder that affects $2-3 \%$ of the world population (Ruscio et al., 2010) and is characterized by the presence of obsessions (intrusive and repetitive thoughts, 
images and/or urges that cause intense anxiety) and/or compulsions (repetitive motor or mental behaviors driven by the subject to reduce the anxiety triggered by obsessions) (APA, 2013). OCD is a very heterogeneous chronic disease that typically establishes during adolescence or young adulthood and available evidence indicates that it has a genetic basis (Taylor, 2011). Additionally, environmental factors such as stress exposure and traumatic life events have been proposed to play an important role in the pathophysiology of the disease (Brander et al., 2016), but the mechanisms remain unknown.

While stress is a highly adaptive response that promotes survival by helping organisms meet the demands of a variety of acute challenges in the short-term (Ursin and Ollf, 1993), prolonged exposure to stress has been shown to disrupt homeostatic mechanisms and lead to maladaptive behavior and neurochemical and morphological adaptations (Sousa, 2012). The normal physiological and behavioral response to stress involves the activation of two major stress pathways: corticotropin releasing factor (CRF) released from the paraventricular nucleus of the hypothalamus, and the autonomic nervous system, which is coordinated by the sympathoadrenal system (Charmandari et al., 2005; McEwen, 1999; Sousa and Almeida, 2012). CRF influences extrahypothalamic regions across the corticostriatal-limbic regions and modulates subjective and behavioral stress responses, while central catecholamine, namely noradrenaline and dopamine, modulate brain motivational pathways, including areas such as the ventral tegmental area, nucleus accumbens and prefrontal cortex (Berridge, 2007; Phan et al., 2005).

Normal cortisol secretion, the main stress hormone in humans, is known to follow a circadian rhythm, with higher basal levels in the early morning that declines during the day, reaching minimal levels in the evening and early night. This daily rhythmicity of cortisol production is regulated by the hypothalamic-pituitary-adrenal (HPA) axis (Oster et al., 2017). An extensive body of research suggests the presence of altered stress response and HPA activity in several negative mood states and many psychiatric disorders such as depression, both in humans and animals (Jarcho et al., 2013; Morris et al., 2012; Reimold et al., 2011). Moreover, prolonged glucocorticoid (GC) levels such as those observed during chronic stress exposure have been proposed to contribute to the development of various disease states, including major depression, schizophrenia, anxiety disorders and addictive disorders (Aguilera, 1994; Bremner et al., 1996; Jarcho et al., 2013; Herman et al., 2012; Nasca et al., 2015; Sinha, 2008; Walker and Diforio, 1997). Likewise, these observations seem to be relevant in OCD pathology given findings showing stressful events may not only precede OCD symptom onset (Toro et al., 1992) but also contribute to exacerbated symptomatology at times of acute stress exposure (Findley et al., 2003). Additionally, it has also been shown that obsessions, a core feature of the disease, increase distress and may thus set in motion the physiological stress response, with consequent increased HPA axis activity. Of note, it has been observed that perceived stress in OCD patients has been correlated with obsessive symptoms (Morgado et al., 2013).

Nonetheless, impaired HPA axis activity and increased circulating levels of cortisol are not consistently found across OCD studies. While some authors report increased cortisol levels in OCD patients (Morgado et al., 2013; Kluge et al., 2007), others have found normal levels compared to control subjects (Atmaca et al., 2005; Kawano et al., 2013). These conflicting results may arise from multiple causes, most importantly methodological differences in the sampling and measurement procedures, selection of participants, disease severity, medication status or even duration of illness, to name a few. It is also important to bear in mind that it remains unclear whether disrupted GC rhythm is the cause or a consequence of the disease.

In an attempt to tackle these issues and clarify whether differences in cortisol levels are present in OCD patients compared to the healthy population, we developed this systematic review and meta-analysis.

\section{Experimental procedures}

This systematic review and meta-analysis was elaborated according to the PRISMA statement (Moher et al., 2009), including search strategy, selection criteria, data extraction and data analysis.

\subsection{Literature search}

The literature search was conducted on PubMed (http: //www.pubmed.gov) using a combination of the following terms: OCD, Obsessive Compulsive Disorder and Cortisol. The search was conducted on August 12, 2016 (updated on May 15, 2017) without any filters. Only original research, experimental studies, were included in this analysis. Reviews, comments, as well as unpublished studies were not considered.

The papers were included if they followed the inclusion criteria: the study was written in English; patients were submitted to standardized diagnostic criteria [Statistical Manual of Mental Disorder, Fourth Edition (DSM-IV) or the International Classification of Disorders 10 (ICD-10)]; data for cortisol levels were presented separately for OCD patients and healthy controls.

\subsection{Data extraction and management}

All papers were screened at the title and abstract levels. Those selected, based on this initial screening were submitted to full-text analysis. This evaluation was conducted by the first author (JL). In the cases where it was not clear whether the manuscript fulfilled the inclusion criteria, a co-author of the manuscript (PSM) was consulted. In the selected papers, the following information was extracted: OCD diagnosis method, sample size, proportion of male and female individuals, range and mean age, cortisol collection period (all day; morning, afternoon, or night), methodology of cortisol assessment (i.e., single versus average of multiple timepoints), qualitative assessment of the comparison of mean cortisol levels between OCD patients and healthy controls. When available, statistical measures were extracted in order to estimate the effect sizes for individual studies.

\subsection{Data analysis}

Studies in which the necessary measures for conducting the meta-analysis could not be calculated were included only in the systematic review. For the remaining, Cohen's $d$ (and the associated variance) was estimated as a measure of effect size (and the 
deviation measure). Cohen's $d$ was computed using the formula:

$$
d=\frac{M_{O C D}-M_{H C}}{S D_{\text {difference }}}
$$

where $M_{O C D}$ and $M_{H C}$ correspond to the average values for the OCD and healthy control groups, respectively; $S D_{\text {difference }}$ pertains to the standard deviation of the difference. The computation of the 95\% confidence interval for the effect-size was calculated as:

$$
\operatorname{var}_{d}=\frac{1}{n}+
$$

In the presence of significant (Cochran $Q$-test with a significance level of $p<0.10$ ) (Higgins and Green, 2008) and/or high levels $\left(I^{2}>75 \%\right)$ of heterogeneity, a random-effects model (the Restricted Maximum-Likelihood method) was used to calculate the summary of pooled estimates. Otherwise, a fixed-effects model (the Mantel-Haenszel method) was performed. To examine the potential causes of between-studies' heterogeneity, we searched for studies with extreme effect sizes (i.e., outliers). A study was identified as an outlier if its confidence interval did not overlap with the pooled confidence interval. In such cases, it is likely that this individual study is not part of the population of the pooled effect size. Leave-one-out (sensitivity) analysis was conducted with the goal of assessing the impact of individual studies in the overall effects. To account for the influence of cortisol collection period (all day; morning, 7:00 a.m. $-00: 30$ p.m.; afternoon, 12:30 p.m.-20 p.m.; night, 20 p.m. -7 a.m.), methodology (serum, urinary and salivary) and number of timepoints in which cortisol was assessed (i.e., single versus average of multiple timepoints) on the overall estimates, moderator meta-analyses were conducted. The presence of potential publication bias was examined through the visual inspection of funnel plot asymmetry, and statistically tested using the rank correlation method from Begg and Mazumdar ( $p<0.05$ represents statistically significant publication bias). The analytical procedures were implemented using Metafor package in R software (Viechtbauer, 2010). The dataset and code supporting the statistical analysis is available at the Open Science Framework (https://osf.io/pc4vf).

\section{Results}

\subsection{Study selection}

The literature search found 124 records. After the screening process, 25 records were selected for full-text review, from which 18 were included in the systematic review. One of these manuscripts presented the findings for two independent samples (Kawano et al., 2013) and, therefore, 19 studies were included in this work. Experiments in which the necessary measures for conducting the meta-analysis (quantitative synthesis) could not be calculated $(n=1)$ (Lucey et al., 1993), were included only in the systematic review (qualitative synthesis) (Fig. 1).

\subsection{Systematic review findings}

The characterization of individual studies is summarized in Tables 1 and 2. Among the 19 studies, 12 used adult samples (>18 years old), one included subjects over 16 years, one had an age range between 7 and 17 years and one included subjects aged 6-12 years old. For the remaining four studies, no information regarding samples' age was presented.
The sample size ranged from 14 to 359 participants. For all the studies, the OCD diagnosis was established according to the DSM (Statistical Manual of Mental Disorder, Third or Fourth Editions). OCD severity was evaluated in 16 studies, with 7 providing information on obsession and compulsion subscales score, and duration of illness was recorded in 11 studies. Additional assessment on other symptoms such as anxiety or depression, was performed in 12 studies, with various instruments being used. Information on medication status was stated in all but one study, with one including only naïve patients, 12 involving drug-free status at the time of the experiments, and 4 including currently medicated patients. Only 5 studies were performed with OCD patients with other psychiatric comorbidities.

Regarding cortisol collection periods, three studies examined cortisol levels during one complete day, eight performed collections during the morning, six in the afternoon, one in the night, whereas one did not report the period in which the cortisol was collected. Regarding the methodology of cortisol assessment, the majority of studies $(n=14)$ implemented a single measurement; for the remaining $(n=5)$, the average of multiple measurements was considered. Cortisol was quantified in the serum (13 studies), saliva (five studies) or urine (one study).

In 12 out of the 19 studies, cortisol levels were found to be significantly higher in OCD patients compared with controls but in seven studies no differences were found between groups.

\subsection{Meta-analytic results}

\subsubsection{Global analysis}

Significant heterogeneity among studies was verified $\left(Q_{(17)}=50.43, p<0.001 ; I^{2}=66.3 \%\right)$ and consequently a Random-Effects model was selected. Overall, differences in cortisol levels were observed in OCD patients relative to the control group $\left(d=0.76, \mathrm{Cl}_{95 \%}=[0.47,1.05], p<0.001\right)$, with $O C D$ patients presenting significantly higher cortisol levels compared with the control group. The overall effect is graphically represented on the forest plot (Fig. 2).

\subsubsection{Sensitivity analysis}

One study was identified as a meta-analytic outlier, given that its confidence interval did not overlap with the confidence interval for the pooled estimate. Nevertheless, the exclusion of this study did not affect the significance of the between-studies' pooled estimates $\left(d=0.69, \mathrm{Cl}_{95 \%}=[0.42\right.$, 0.96]). In addition, it was observed that the exclusion of a single study did not yield significant changes in overall effects (Fig. S1).

\subsubsection{Subgroup analysis}

It was observed that the pooled effects were statistically significant for studies with single measurement $(d=0.62$, $\left.\mathrm{Cl}_{95 \%}=[0.42,0.96]\right)$ and for studies with multiple cortisol assessments $\left(d=1.16, \mathrm{Cl}_{95 \%}=[0.67,1.65]\right)$. Nevertheless, the comparison of the overall effects for each subgroup metaanalysis revealed a trend for lower higher standardized effects for the pooling of studies with multiple assessments $(z=1.79, p=0.073)$. It was observed that the cortisol collection period yielded significant subgroup differences 
Table 1 Characteristics of studies included in the systematic review and meta-analysis.

\begin{tabular}{|c|c|c|c|c|c|c|c|c|c|}
\hline \multirow[t]{2}{*}{ Study } & \multicolumn{2}{|c|}{ Participants } & \multicolumn{2}{|c|}{$\begin{array}{l}\text { Age range, years } \\
\text { (mean } \pm \text { SD) }\end{array}$} & \multirow[t]{2}{*}{$\begin{array}{l}\text { Evaluation } \\
\text { procedures }\end{array}$} & \multirow[t]{2}{*}{$\begin{array}{l}\text { Type of sample (time } \\
\text { of collection) }\end{array}$} & \multicolumn{3}{|c|}{ Results/Conclusions } \\
\hline & $\begin{array}{l}\text { OCD } \\
\text { group }\end{array}$ & $\begin{array}{l}\text { CONT } \\
\text { group }\end{array}$ & OCD group & CONT group & & & OCD group & CONT group & Comparison \\
\hline $\begin{array}{l}\text { Bastani } \\
\text { (1990) }\end{array}$ & $\begin{array}{l}N=17 \\
(9 F, 8 M)\end{array}$ & $\begin{array}{l}N=9 \\
(4 \mathrm{~F}, 5 \mathrm{M})\end{array}$ & $\begin{array}{l}F: 24-45 \\
(35.2 \pm 6.8) \\
M: 24-40 \\
(31.0 \pm 5.5)\end{array}$ & $\begin{array}{l}\text { F: } 25-55 \\
(35 \pm 13.8) \\
M: 20-45 \\
(34.8 \pm 9.9)\end{array}$ & $\begin{array}{l}\text { Structured interview } \\
\quad \text { (DSM-III-R) } \\
\text { Y-BOCS } \\
\text { HDRS }\end{array}$ & Blood (morning) & $\begin{array}{l}9.0 \pm 3.0 \\
\mu \mathrm{g} / \mathrm{dL}\end{array}$ & $\begin{array}{l}8.63 \pm 4.39 \\
\mu \mathrm{g} / \mathrm{dL}\end{array}$ & $\begin{array}{l}t=0.36 \\
d f=24 \\
p=0.72\end{array}$ \\
\hline $\begin{array}{l}\text { Gehris et al. } \\
\text { (1990) }\end{array}$ & $\begin{array}{l}N=17 \\
(12 \mathrm{~F}, 5 \mathrm{M})\end{array}$ & $\begin{array}{l}N=25 \\
(14 \mathrm{~F}, 11 \mathrm{M})\end{array}$ & $\begin{array}{l}20-62 \\
(35.6 \pm 10.3)\end{array}$ & $\begin{array}{l}21-60 \\
(37.6 \pm 8.4)\end{array}$ & $\begin{array}{l}\text { Structured interview } \\
\quad \text { (DSM-III-R) } \\
\text { Y-BOCS } \\
\text { NIMH-GOCS } \\
\text { HDRS }\end{array}$ & Urine $(24 \mathrm{~h})$ & $\begin{array}{l}24.6 \pm 9.3 \\
\mu \mathrm{g} / \mathrm{g}-\mathrm{cr}\end{array}$ & $\begin{array}{l}17.0 \pm 5.6 \\
\mu \mathrm{g} / \mathrm{g}-\mathrm{cr}\end{array}$ & $\begin{array}{l}t=3.02 ; \\
\mathrm{df}=24.94 ; \\
p<0.006\end{array}$ \\
\hline $\begin{array}{l}\text { Weizman } \\
\text { et al. } \\
\text { (1990) }\end{array}$ & $\begin{array}{l}N=13 \\
(10 F, 3 M)\end{array}$ & $\begin{array}{l}N=20 \\
(15 F, 5 M)\end{array}$ & $\begin{array}{l}16-48 \\
(32.9 \pm 8.1)\end{array}$ & $\begin{array}{l}17-47 \\
(32.9 \pm 8.3)\end{array}$ & $\begin{array}{l}\text { Semi-structured } \\
\text { interview (DSM-III) } \\
\text { Y-BOCS } \\
\text { HDRS }\end{array}$ & Blood (morning) & $\begin{array}{l}13.6 \pm 2.1 \\
\mu \mathrm{g} / \mathrm{dL}(N=13)\end{array}$ & $\begin{array}{l}10.5 \pm 1.1 \\
\mu \mathrm{g} / \mathrm{dL}(N=17)\end{array}$ & NS \\
\hline $\begin{array}{l}\text { Lesch et al. } \\
\text { (1991) }\end{array}$ & $\begin{array}{l}N=12 \\
(4 \mathrm{~F}, 8 \mathrm{M})\end{array}$ & $\begin{array}{l}N=22 \\
(8 F, 14 M)\end{array}$ & $35,8 \pm 11.7$ & $34,8 \pm 12.7$ & $\begin{array}{l}\text { Structured interview } \\
\quad \text { (DSM-III-R) } \\
\text { Y-BOCS } \\
\text { HDRS } \\
\text { STAI }\end{array}$ & Blood (afternoon) & No differences & een $O C D$ and $C O N$ & ubjects \\
\hline $\begin{array}{l}\text { Lucey et al. } \\
\qquad(1993)\end{array}$ & $\begin{array}{l}N=8 \\
(1 F, 7 M)\end{array}$ & $\begin{array}{l}N=8 \\
(1 F, 7 M)\end{array}$ & $\begin{array}{l}22-39 \\
(31 \pm 6)\end{array}$ & $\begin{array}{l}26-45 \\
(31 \pm 6)\end{array}$ & $\begin{array}{l}\text { Semi-structured } \\
\text { interview (DSM-III) } \\
\text { Y-BOCS } \\
\text { CGIS }\end{array}$ & $\begin{array}{c}\text { Blood } \\
\text { (morning) }\end{array}$ & $\begin{array}{l}\text { Baseline cortisc } \\
\text { patients and }\end{array}$ & $\begin{array}{l}\text { els did not differ } \\
\text { ols }\end{array}$ & ween OCD \\
\hline $\begin{array}{l}\text { Monteleone } \\
\text { et al. } \\
\text { (1994) }\end{array}$ & $\begin{array}{l}N=13 \\
(4 \mathrm{~F}, 9 \mathrm{M})\end{array}$ & $\begin{array}{l}N=13 \\
(4 \mathrm{~F}, 8 \mathrm{M})\end{array}$ & $\begin{array}{l}22-59 \\
(31.0 \pm 11.7)\end{array}$ & $\begin{array}{l}22-60 \\
(31.3 \pm 11.4)\end{array}$ & $\begin{array}{l}\text { Structured interview } \\
\quad \text { (DSM-III-R) } \\
\text { Y-BOCS } \\
\text { HDRS }\end{array}$ & $\begin{array}{l}\text { Blood } \\
\text { (multiple timepoints } \\
20 \text { p.m.-16 p.m.) }\end{array}$ & $\begin{array}{c}\text { Mean circadian } \\
\text { patients simi } \\
\text { levels. Plasm } \\
24 \text { p.m., } 01 \text { a } \\
08 \text { a.m. signi } \\
(p<0.05)\end{array}$ & $\begin{array}{l}\text { le of plasma cort } \\
\text { that of controls } \\
\text { tisol levels at } 20 \\
02 \text { a.m., } 03 \text { a.m. } \\
\text { cly higher than in }\end{array}$ & $\begin{array}{l}\text { in } O C D \\
\text { at higher } \\
\text { n., } 22 \text { p.m., } \\
4 \text { a.m. and } \\
\text { rmal subjects }\end{array}$ \\
\hline $\begin{array}{l}\text { Monteleone } \\
\text { et al. } \\
\text { (1995) }\end{array}$ & $\begin{array}{l}N=7 \\
(2 F, 5 M)\end{array}$ & $\begin{array}{l}N=7 \\
(4 F, 9 M)\end{array}$ & $\begin{array}{l}22-59 \\
(33.7 \pm 14.7)\end{array}$ & $\begin{array}{l}22-60 \\
(32.7 \pm 12.1)\end{array}$ & $\begin{array}{l}\text { Structured interview } \\
\quad \text { (DSM-III-R) } \\
\text { Y-BOCS } \\
\text { CGIS } \\
\text { HDRS }\end{array}$ & $\begin{array}{l}\text { Blood } \\
\text { (multiple timepoints } \\
20 \text { p.m.-16 p.m.) }\end{array}$ & $\begin{array}{l}\text { Increased circac } \\
\text { OCD patients }\end{array}$ & $\begin{array}{l}\text { ecretion of cortis } \\
\text { ive to matched cc }\end{array}$ & $\begin{array}{l}(p<0.01) \text { in } \\
\text { rols }\end{array}$ \\
\hline
\end{tabular}




\begin{tabular}{|c|c|c|c|c|c|c|c|c|c|}
\hline \multirow[t]{2}{*}{ Study } & \multicolumn{2}{|l|}{ Participants } & \multicolumn{2}{|c|}{$\begin{array}{l}\text { Age range, years } \\
(\text { mean } \pm S D)\end{array}$} & \multirow[t]{2}{*}{$\begin{array}{l}\text { Evaluation } \\
\text { procedures }\end{array}$} & \multirow[t]{2}{*}{$\begin{array}{l}\text { Type of sample (time } \\
\text { of collection) }\end{array}$} & \multicolumn{3}{|c|}{ Results/Conclusions } \\
\hline & $\begin{array}{l}\text { OCD } \\
\text { group }\end{array}$ & $\begin{array}{l}\text { CONT } \\
\text { group }\end{array}$ & $O C D$ group & CONT group & & & $O C D$ group & CONT group & Comparison \\
\hline $\begin{array}{l}\text { Monteleone } \\
\text { et al. } \\
\text { (1997) }\end{array}$ & $\begin{array}{l}N=20 \\
(10 F, 10 M)\end{array}$ & $\begin{array}{l}N=20 \\
(10 F, 10 M)\end{array}$ & $\begin{array}{l}18-64 \\
(32.7 \pm 12.3)\end{array}$ & $\begin{array}{l}18-64 \\
(34.6 \pm 9.5)\end{array}$ & $\begin{array}{l}\text { Structured interview } \\
\text { (DSM-IV) } \\
\text { Y-BOCS } \\
\text { HDRS }\end{array}$ & Blood (morning) & \multicolumn{3}{|c|}{$\begin{array}{l}\text { Baseline plasma cortisol significantly higher in OCD } \\
\text { patients }(p<0.05)\end{array}$} \\
\hline $\begin{array}{l}\text { Atmaca } \\
\text { et al. } \\
(2005)\end{array}$ & $\begin{array}{l}N=44 \\
(27 F, 17 M)\end{array}$ & $\begin{array}{l}N=30 \\
(18 \mathrm{~F}, 12 \mathrm{M})\end{array}$ & $\begin{array}{l}18-49 \\
(28.2 \pm 4.9)\end{array}$ & $\begin{array}{l}19-47 \\
(26.5 \pm 6.6)\end{array}$ & $\begin{array}{l}\text { Semi-structured } \\
\text { interview (DSM-IV) } \\
\text { Y-BOCS } \\
\text { HDRS }\end{array}$ & Blood (morning) & \multicolumn{3}{|c|}{$\begin{array}{l}\text { Pure OCD patients have comparable cortisol levels } \\
\text { compared to controls }(8.8 \pm 2.7 \mathrm{mg} / \mathrm{dL} \text { and } \\
9.4 \pm 3.3 \mathrm{mg} / \mathrm{dL} \text {, respectively, } p>0.05) \text {, while } O C D \\
\text { patients with comorbid depression have higher } \\
\text { cortisol than pure } O C D \text { and control subjects } \\
(12.5 \pm 3.8 \mathrm{mg} / \mathrm{dL}, p<0.01)\end{array}$} \\
\hline $\begin{array}{l}\text { Kluge et al. } \\
\quad(2007)\end{array}$ & $\begin{array}{l}N=9 \text { inpa- } \\
\text { tients } \\
(2 F, 7 M)\end{array}$ & $\begin{array}{l}N=9 \\
(2 F, 7 M)\end{array}$ & $\begin{array}{l}20-46 \\
(31.8 \pm 9.3)\end{array}$ & $\begin{array}{l}21-46 \\
(31.6 \pm 9.1)\end{array}$ & $\begin{array}{l}\text { Structured interview } \\
\text { (DSM-IV) } \\
\text { Y-BOCS } \\
\text { HDRS }\end{array}$ & $\begin{array}{l}\text { Blood } \\
\text { (multiple timepoints } \\
23 \text { p.m.-07 a.m.) }\end{array}$ & \multicolumn{3}{|c|}{$\begin{array}{l}\text { Nocturnal plasma cortisol levels significantly elevated } \\
\text { in OCD patients relative to controls }(p<0.005)\end{array}$} \\
\hline $\begin{array}{l}\text { Gustafsson } \\
\text { et al. } \\
\text { (2008) }\end{array}$ & $\begin{array}{l}N=23 \\
(13 F, 10 M)\end{array}$ & $N=336$ & $\begin{array}{l}\text { 9-17 } \\
\text { (median 13) }\end{array}$ & $\begin{array}{l}\text { 6-12 } \\
\text { (median 9) }\end{array}$ & $\begin{array}{l}\text { Semi-structured } \\
\quad \text { interview (DSM-IV) } \\
\text { WISC } \\
\text { KSADS-PL } \\
\text { CY-BOCS } \\
\text { CGIS } \\
\text { C-GAS }\end{array}$ & $\begin{array}{l}\text { Saliva } \\
\text { (early-morning, } \\
\text { late-morning, } \\
\text { night) }\end{array}$ & $\begin{array}{l}8.3 \pm 2.9 \mathrm{nmol} / \mathrm{L} \\
4.1 \pm 1.8 \mathrm{nmol} / \mathrm{L} \\
1.0 \pm 0.5 \mathrm{nmol} / \mathrm{L}\end{array}$ & $\begin{array}{l}5.7 \pm 4.2 \mathrm{nmol} / \mathrm{L} \\
3.5 \pm 2.2 \mathrm{nmol} / \mathrm{L} \\
1.3 \pm 0.5 \mathrm{nmol} / \mathrm{L}\end{array}$ & $\begin{array}{l}p=0.005 \\
p=0.180 \\
p=0.400\end{array}$ \\
\hline $\begin{array}{l}\text { Fluitman } \\
\text { et al. } \\
\text { (2010) }\end{array}$ & $\begin{array}{l}N=10 \\
(9 F, 1 M)\end{array}$ & $\begin{array}{l}N=10 \\
(8 F, 2 M)\end{array}$ & $34.6 \pm 9.0$ & $32.5 \pm 7.9$ & $\begin{array}{l}\text { Structured interview } \\
\text { (DSM-IV) } \\
\text { Y-BOCS }\end{array}$ & Blood (morning) & \multicolumn{3}{|c|}{$\begin{array}{l}\text { Plasma cortisol levels significantly higher in OCD } \\
\text { patients relative to controls }(p=0.015)\end{array}$} \\
\hline $\begin{array}{l}\text { Lord et al. } \\
\qquad(2011)\end{array}$ & $\begin{array}{l}N=8 \\
(\mathrm{ppOCD})\end{array}$ & $\begin{array}{l}N=10 \\
(\mathrm{ppCONT})\end{array}$ & $\begin{array}{l}>18 \text { years } \\
\text { (within } 6 \text { mon }\end{array}$ & רs postpartum) & $\begin{array}{l}\text { Structured interview } \\
\quad \text { (DSM-IV) } \\
\text { CIDI-VENUS } \\
\text { Y-BOCS } \\
\text { EPDS } \\
\text { STAI }\end{array}$ & Saliva (afternoon) & \multicolumn{3}{|c|}{$\begin{array}{l}\text { Basal salivary cortisol levels significantly elevated in } \\
\text { OCD compared to CONT }\end{array}$} \\
\hline
\end{tabular}


Table 1 (continued)

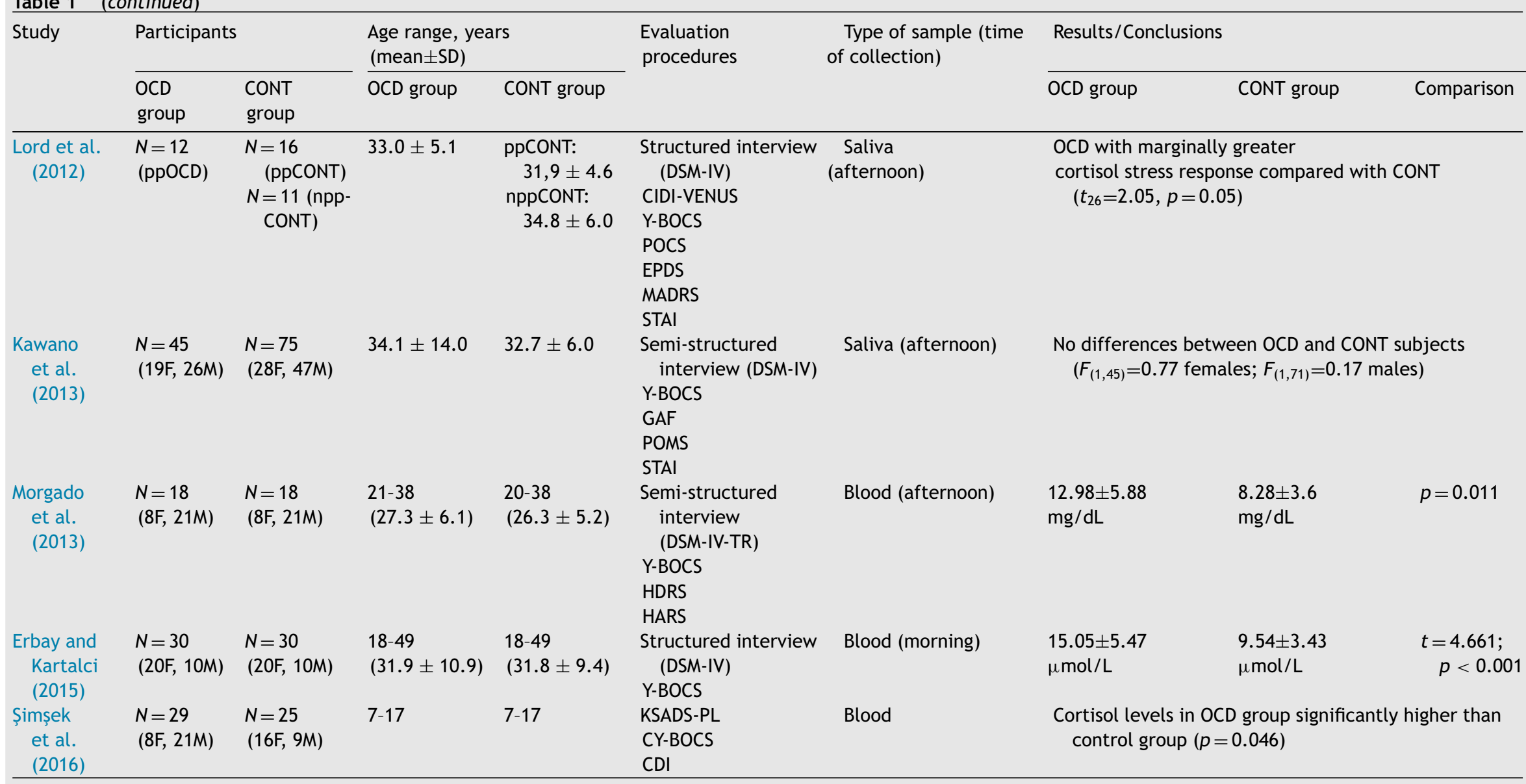

ADHD, Attention-deficit hyperactivity disorder; CDI, Children's Depression Inventory; C-GAS, Children's Global Assessment Scale; CGIS, Clinical Global Impression Scale; CIDI-VENUS, Composite International Diagnostic Interview for Women; CONT, control; CY-BOCS, Children's Yale-Brown Obsessive-Compulsive Scale; DSM, Diagnostic and Statistical Manual of Mental Disorders; EPDS, Edinburgh Postnatal Depression Scale; F, Female; GAD, Generalized Anxiety Disorder; GAF, Global Assessment of Functioning; HARS, Hamilton Anxiety Rating Scale; HDRS, Hamilton Depression Rating Scale; K-SADS-PL, Kiddie Schedule for Affective Disorders and Schizophrenia, Present and Lifetime Version; M, Male; MADRS, Montgomery-Ásberg Depression Rating Scale; MDD, Major Depressive Disorder; NIMH-GOCS; NIMH Global Obsessive-Compulsive Scale; OCD, Obsessive-Compulsive Disorder; PD, Panic disorder; POCS, Perinatal Obsessive-Compulsive Scale; POMS, Profile of Mood State; SD, standard deviation; SP, specific phobia; STAI, State-Trait Anxiety Inventory; TS, Tourette's Syndrome; WISC, Wechsler Intelligence Scale for Children; Y-BOCS, Yale-Brown Obsessive-Compulsive Scale. 
Table 2 Clinical characterization of OCD samples of studies included in the systematic review and meta-analysis.

\begin{tabular}{|c|c|c|c|c|c|c|}
\hline Study & OCD assessment & $\begin{array}{l}\text { Symptom severity } \\
(\text { mean } \pm \text { SD) }\end{array}$ & $\begin{array}{l}\text { Duration of illness } \\
\text { (years) }\end{array}$ & Medication status (N) & Comorbidities (N) & $\begin{array}{l}\text { Other assessments } \\
(\text { mean } \pm \text { SD })\end{array}$ \\
\hline Bastani (1990) & $\begin{array}{l}\text { Structured interview } \\
\text { (DSM-III-R) } \\
\text { Y-BOCS } \\
\mathrm{MOCl}\end{array}$ & $\begin{array}{l}\text { Y-BOCS }(22.81 \pm 6.13) \\
\mathrm{MOCl}(16.76 \pm 6.13)\end{array}$ & - & $\begin{array}{l}\text { Drug-free for at least } \\
1 \text { month }\end{array}$ & No & HDRS (15.87 \pm 6.05$)$ \\
\hline $\begin{array}{l}\text { Gehris et al. } \\
(1990)\end{array}$ & $\begin{array}{l}\text { Structured interview } \\
\text { (DSM-III-R) } \\
\text { Y-BOCS } \\
\text { NIMH-GOCS }\end{array}$ & $\begin{array}{l}\text { Y-BOCS (NA) } \\
\text { NIMH-GOCS (NA) }\end{array}$ & $11.5 \pm 6.4$ & Drug-free 4 weeks prior & No & HDRS (NA) \\
\hline $\begin{array}{l}\text { Weizman et al. } \\
\text { (1990) }\end{array}$ & $\begin{array}{l}\text { Semi-structured interview } \\
\text { (DSM-III) }\end{array}$ & - & - & Drug-free at least 1 year & No & - \\
\hline Lesch et al. (1991) & $\begin{array}{l}\text { Structured interview } \\
\text { (DSM-III-R) } \\
\text { Y-BOCS }\end{array}$ & Y-BOCS $(22.5 \pm 6.2)$ & $6.9 \pm 7.8$ & $\begin{array}{l}\text { Drug-free at least } \\
21 \text { days }\end{array}$ & $\begin{array}{l}\text { Mild depression ( } \mathrm{N} \\
\text { not indicated) }\end{array}$ & $\begin{array}{l}\text { HDRS }(6.5 \pm 4.7) \\
\text { STAI-S }(46.1 \pm 11.8) \\
\text { STAI-T }(43.9 \pm 12.1)\end{array}$ \\
\hline Lucey et al. (1993) & $\begin{array}{l}\text { Semi-structured interview } \\
\text { (DSM-III) } \\
\text { Y-BOCS } \\
\text { CGIS }\end{array}$ & $\begin{array}{l}\text { Y-BOCS }(24.0 \pm 8.2) \\
\text { CGIS (NA) }\end{array}$ & At least 1 year & $\begin{array}{l}\text { Drug-free at least } \\
3 \text { months }\end{array}$ & No & - \\
\hline $\begin{array}{l}\text { Monteleone et al. } \\
\text { (1994) }\end{array}$ & $\begin{array}{l}\text { Structured interview } \\
\text { (DSM-III-R) } \\
\text { Y-BOCS }\end{array}$ & $\begin{array}{l}\text { Y-BOCS } \\
\text { - Total }(25.8 \pm 5.1) \\
\text { - Obsession }(11.0 \pm 3.3) \\
\text { - Compulsion } \\
\quad(14.7 \pm 2.8)\end{array}$ & $6.4 \pm 4.9$ & $\begin{array}{l}\text { Drug-free at least } \\
3 \text { months }\end{array}$ & No & HDRS $(10.0 \pm 3.9)$ \\
\hline $\begin{array}{l}\text { Monteleone et al. } \\
\text { (1995) }\end{array}$ & $\begin{array}{l}\text { Structured interview } \\
\quad \text { (DSM-III-R) } \\
\text { Y-BOCS } \\
\text { CGIS }\end{array}$ & $\begin{array}{l}\text { Y-BOCS } \\
\text { - Total }(27.5 \pm 6.6) \\
\text { - Obsession }(12.7 \pm 2.5) \\
\text { - Compulsion } \\
\quad(14.8 \pm 6.4) \\
\text { GCIS (NA) }\end{array}$ & $57.4 \pm 32$ months & $\begin{array}{l}\text { Drug-free at least } \\
3 \text { months }\end{array}$ & No & HDRS $(9.7 \pm 3.4)$ \\
\hline $\begin{array}{l}\text { Monteleone et al. } \\
\text { (1997) }\end{array}$ & $\begin{array}{l}\text { Structured interview } \\
\text { (DSM-IV) } \\
\text { Y-BOCS }\end{array}$ & $\begin{array}{l}\text { Y-BOCS } \\
\text { - Total }(26.4 \pm 6.3) \\
\text { - Obsession }(13.6 \pm 3.1) \\
\text { - Compulsion } \\
\quad(12.8 \pm 4.0)\end{array}$ & $5.6 \pm 5.0$ & $\begin{array}{l}\text { Drug-free at least } \\
3 \text { months }\end{array}$ & No & HDRS $(13.1 \pm 7.9)$ \\
\hline $\begin{array}{l}\text { Atmaca et al. } \\
\text { (2005) }\end{array}$ & $\begin{array}{l}\text { Semi-structured interview } \\
\text { (DSM-IV) } \\
\text { Y-BOCS }\end{array}$ & $\begin{array}{l}\text { OCD+D }(24.1 \pm 4.3) \\
\text { OCD-D }(21.1 \pm 3.9)\end{array}$ & - & Drug-free 2 weeks prior & $\begin{array}{l}\text { MDD (15) } \\
\text { Dystimic disorder } \\
(2)\end{array}$ & $\begin{array}{l}\text { HDRS } \\
\text { - OCD+D }(14.2 \pm 0,47) \\
\text { - OCD-D }(7.2 \pm 2.2)\end{array}$ \\
\hline
\end{tabular}




\begin{tabular}{|c|c|c|c|c|c|c|}
\hline Study & OCD assessment & $\begin{array}{l}\text { Symptom severity } \\
\text { (mean } \pm \text { SD) }\end{array}$ & $\begin{array}{l}\text { Duration of illness } \\
\text { (years) }\end{array}$ & Medication status $(\mathrm{N})$ & Comorbidities (N) & $\begin{array}{l}\text { Other assessments } \\
(\text { mean } \pm \text { SD })\end{array}$ \\
\hline Kluge et al. (2007) & $\begin{array}{l}\text { Structured interview } \\
\text { (DSM-IV) } \\
\text { Y-BOCS }\end{array}$ & Y-BOCS $(27.3 \pm 4.3)$ & $7.0 \pm 8.8$ & $\begin{array}{l}\text { Drug-free at least } 7 \text { days } \\
\text { (Fluoxetine-free at least } \\
6 \text { weeks) }\end{array}$ & No & HDRS $(13.3 \pm 1.9)$ \\
\hline $\begin{array}{l}\text { Gustafsson et al. } \\
\qquad(2008)\end{array}$ & $\begin{array}{l}\text { Semi-structured interview } \\
\quad \text { (DSM-IV) } \\
\text { KSADS-PL } \\
\text { CY-BOCS } \\
\text { CGIS }\end{array}$ & $\begin{array}{l}\text { CY-BOCS (NA) } \\
\text { CGIS (NA) }\end{array}$ & - & $\begin{array}{l}\text { With current medication } \\
\qquad(N=4)\end{array}$ & $\begin{array}{l}\text { ADHD (7) } \\
\text { Tics (6) } \\
\text { SP (5) } \\
\text { TS (5) } \\
\text { MDD (3) } \\
\text { Dystimia (2) } \\
\text { Other (5) }\end{array}$ & $\begin{array}{l}\text { C-GAS (NA) } \\
\text { WISC (NA) }\end{array}$ \\
\hline $\begin{array}{l}\text { Fluitman et al. } \\
\qquad(2010)\end{array}$ & $\begin{array}{l}\text { Structured interview } \\
\text { (DSM-IV) } \\
\text { Y-BOCS }\end{array}$ & $\begin{array}{l}\text { Y-BOCS } \\
\text { - Total }(30.2 \pm 5.4) \\
\text { - Obsession }(14.0 \pm 3.1) \\
\text { - Compulsion } \\
\quad(16.2 \pm 2.8)\end{array}$ & 21 years & $\begin{array}{l}\text { Non-medicated }(N=5) \\
\text { Paroxetine, } 60 \mathrm{mg} / \mathrm{d} \\
(N=3) \\
\text { Venlafaxine, } 150 \mathrm{mg} / \mathrm{d} \\
(N=1) \\
\text { Citalopram, } 60 \mathrm{mg} / \mathrm{d} \\
(N=1)\end{array}$ & - & - \\
\hline Lord et al. (2011) & $\begin{array}{l}\text { Structured interview } \\
\text { (DSM-IV) } \\
\text { CIDI-VENUS } \\
\text { Y-BOCS }\end{array}$ & Y-BOCS $(15.0 \pm 6.8)$ & - & - & No & $\begin{array}{l}\text { EPDS }(7.9 \pm 2.4) \\
\text { STAI-S }(41.1 \pm 11.7) \\
\text { STAI-T }(42.0 \pm 12.8) \\
\text { PSQI }(10.0 \pm 4.5) \\
\text { RSE }(18.0 \pm 6.2) \\
\text { CTQ }(31.5 \pm 7.4)\end{array}$ \\
\hline Lord et al. (2012) & $\begin{array}{l}\text { Structured interview } \\
\quad \text { (DSM-IV) } \\
\text { CIDI-VENUS } \\
\text { Y-BOCS } \\
\text { POCS }\end{array}$ & $\begin{array}{l}\text { Y-BOCS }(14.8 \pm 9.2) \\
\text { POCS }(17.3 \pm 8.7)\end{array}$ & - & $\begin{array}{l}\text { Non-medicated }(N=1) \\
\text { Escitalopram }(N=2) \\
\text { Quetiapine }(N=3) \\
\text { Lorazepam }(N=2) \\
\text { Venlafaxine }(N=1) \\
\text { Paroxetine }(N=1) \\
\text { Sertraline }(N=1) \\
\text { Fluvoxamine }(N=1) \\
\text { Citalopram }(N=2)\end{array}$ & No & $\begin{array}{l}\text { EPDS }(7.9 \pm 3.5) \\
\text { MADRS }(6.1 \pm 3.4) \\
\text { STAI-T }(42.5 \pm 10.4) \\
\text { PSQI }(10.6 \pm 3.8) \\
\text { RSE }(18.3 \pm 5.7) \\
\text { CTQ }(35.5 \pm 10.8)\end{array}$ \\
\hline $\begin{array}{l}\text { Kawano et al. } \\
\text { (2013) }\end{array}$ & $\begin{array}{l}\text { Semi-structured interview } \\
\text { (DSM-IV) } \\
\text { Y-BOCS }\end{array}$ & Y-BOCS $(28.1 \pm 7.5)$ & - & $\begin{array}{l}\text { SSRI }(N=41) \\
\text { TCA }(N=4) \\
\text { (all drug-free } 5 \text { h prior } \\
\text { to study) }\end{array}$ & $\begin{array}{l}\text { PD }(N=11) \\
\text { GAD }(N=3)\end{array}$ & $\begin{array}{l}\text { GAF }(\text { NA) } \\
\text { STAI-S }(53.9 \pm 12.5) \\
\text { STAI-T }(59.1 \pm 13.1) \\
\text { POMS tension/anxiety } \\
\quad(64.0 \pm 12.8) \\
\text { POMS } \\
\quad \text { depression/dejection } \\
(65.8 \pm 11.9) \\
\text { (continued on next page) }\end{array}$ \\
\hline
\end{tabular}




\begin{tabular}{|c|c|c|c|c|c|c|}
\hline Study & OCD assessment & $\begin{array}{l}\text { Symptom severity } \\
\text { (mean } \pm \text { SD) }\end{array}$ & $\begin{array}{l}\text { Duration of illness } \\
\text { (years) }\end{array}$ & Medication status (N) & Comorbidities (N) & $\begin{array}{l}\text { Other assessments } \\
\text { (mean } \pm \text { SD) }\end{array}$ \\
\hline $\begin{array}{l}\text { Morgado et al. } \\
\text { (2013) }\end{array}$ & $\begin{array}{l}\text { Semi-structured interview } \\
\text { (DSM-IV-TR) } \\
\text { Y-BOCS }\end{array}$ & $\begin{array}{l}\text { Y-BOCS } \\
\text { - Total }(25.61 \pm 5.91) \\
\text { - Obsession }(13.5 \pm 3.17) \\
\text { - Compulsion } \\
\quad(12.11 \pm 3.27)\end{array}$ & $5.71 \pm 6.70$ & $\begin{array}{l}\text { SSRI }(N=14) \\
\text { SSRI+TCA }(N=4)\end{array}$ & No & $\begin{array}{l}\text { HDRS }(3.83 \pm 2.54) \\
\text { HARS }(4.33 \pm 3.20) \\
\text { PSS-10 (NA) }\end{array}$ \\
\hline $\begin{array}{l}\text { Erbay and Kartalci } \\
\qquad \text { (2015) }\end{array}$ & $\begin{array}{l}\text { Structured interview } \\
\text { (DSM-IV) } \\
\text { Y-BOCS }\end{array}$ & $\begin{array}{l}\text { Y-BOCS } \\
\text { - Total }(28.30 \pm 5.09) \\
\text { - Obsession }(14.53 \pm 2.31) \\
\text { - Compulsion } \\
\quad(14.10 \pm 2.46)\end{array}$ & $7.87 \pm 6.658$ & $\begin{array}{l}\text { Drug-free at time of } \\
\text { study }\end{array}$ & No & - \\
\hline $\begin{array}{l}\text { Şimșek et al. } \\
\text { (2016) }\end{array}$ & $\begin{array}{l}\text { KSADS-PL } \\
\text { CY-BOCS }\end{array}$ & $\begin{array}{l}\text { CY-BOCS } \\
\text { - Total }(23.6 \pm 6.6) \\
\text { - Obsession }(12.3 \pm 3.4) \\
\text { - Compulsion } \\
\quad(11.3 \pm 3.5)\end{array}$ & $\begin{array}{c}17.9 \pm 18.5 \\
\text { months }\end{array}$ & Naïve patients & $\begin{array}{l}\text { Simple motor tic } \\
\text { disorder }(N=4)\end{array}$ & CDI $(12.8 \pm 6.6)$ \\
\hline
\end{tabular}

ADHD, Attention-deficit hyperactivity disorder; CDI, Children's Depression Inventory; C-GAS, Children's Global Assessment Scale; CGIS, Clinical Global Impression Scale; CIDI-VENUS, Composite International Diagnostic Interview for Women; CONT, control; CTQ, Childhood Trauma Questionnaire; CY-BOCS, Children's Yale-Brown Obsessive-Compulsive Scale; DSM, Diagnostic and Statistical Manual of Mental Disorders; EPDS, Edinburgh Postnatal Depression Scale; F, Female; GAD, Generalized Anxiety Disorder; GAF, Global Assessment of Functioning; HARS, Hamilton Anxiety Rating Scale; HDRS, Hamilton Depression Rating Scale; K-SADS-PL, Kiddie Schedule for Affective Disorders and Schizophrenia, Present and Lifetime Version; M, Male; MADRS, Montgomery-Åsberg Depression Rating Scale; MDD, Major Depressive Disorder; NIMH-GOCS; NIMH Global Obsessive-Compulsive Scale; OCD, Obsessive-Compulsive Disorder; OCD+D, Obsessive-Compulsive Disorder with concurrent Depression; OCD+D, Obsessive-Compulsive Disorder without concurrent Depression; PD, Panic disorder; POCS, Perinatal ObsessiveCompulsive Scale; POMS, Profile of Mood State; PSIQ, Pittsburg Sleep Quality Index; PSS-10, Perceived Stress Scale; RSE, Rosenberg Self-Esteem questionnaire; SD, standard deviation; SP, specific phobia; SSRI, Selective Serotonine Reuptake Inhibitor; STAI, State-Trait Anxiety Inventory; TCA, Tricyclic Anti-Depressant; TS, Tourette's Syndrome; WISC, Wechsler Intelligence Scale for Children; Y-BOCS, Yale-Brown Obsessive-Compulsive Scale. 


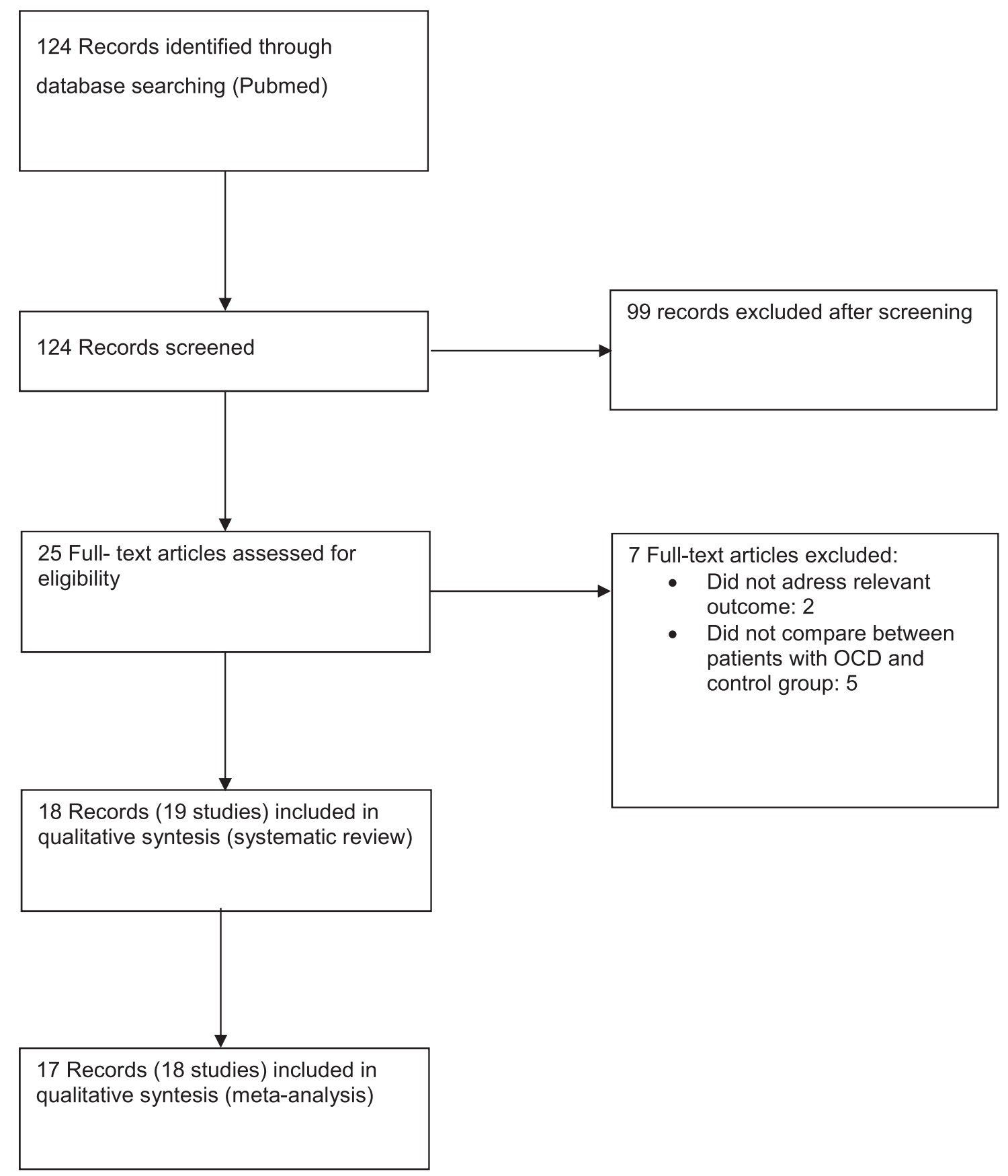

Fig. 1 PRISMA diagram.

$(p=0.032)$. Studies reporting cortisol levels pertaining to the entire day produced the most pronounced results $\left(d=1.28, \mathrm{Cl}_{95 \%}=[0.80,1.77]\right)$, followed by studies reporting measurements during the morning period $(d=0.72$, $\left.\mathrm{Cl}_{95 \%}=[0.26,1.18]\right)$. Lower estimates were found for the group of studies reporting cortisol assessments during the afternoon $(d=0.46)$, for which the lower bound of the confidence interval was close to $0\left(\mathrm{Cl}_{95 \%}=[0.08,0.85]\right)$. Finally, it was noted that the pooled estimates for both the assessment of cortisol on serum $\left(d=0.85, \mathrm{Cl}_{95 \%}=[0.45,1.26]\right)$ and using other approaches (encompassing salivary and urinary cortisol; $\left.d=0.48, \mathrm{Cl}_{95 \%}=[0.11,0.86]\right)$ were statistically significant estimates. Even though the magnitude of the estimates was strong for the group of studies assessing cortisol on the serum, while being of moderate magnitude for the remaining approaches, the difference between the estimates was not statistically significant $(p=0.191)$.

\subsubsection{Publication bias}

The funnel plot revealed a considerable asymmetry (Fig. 3) between studies, suggesting a potential publication bias. 


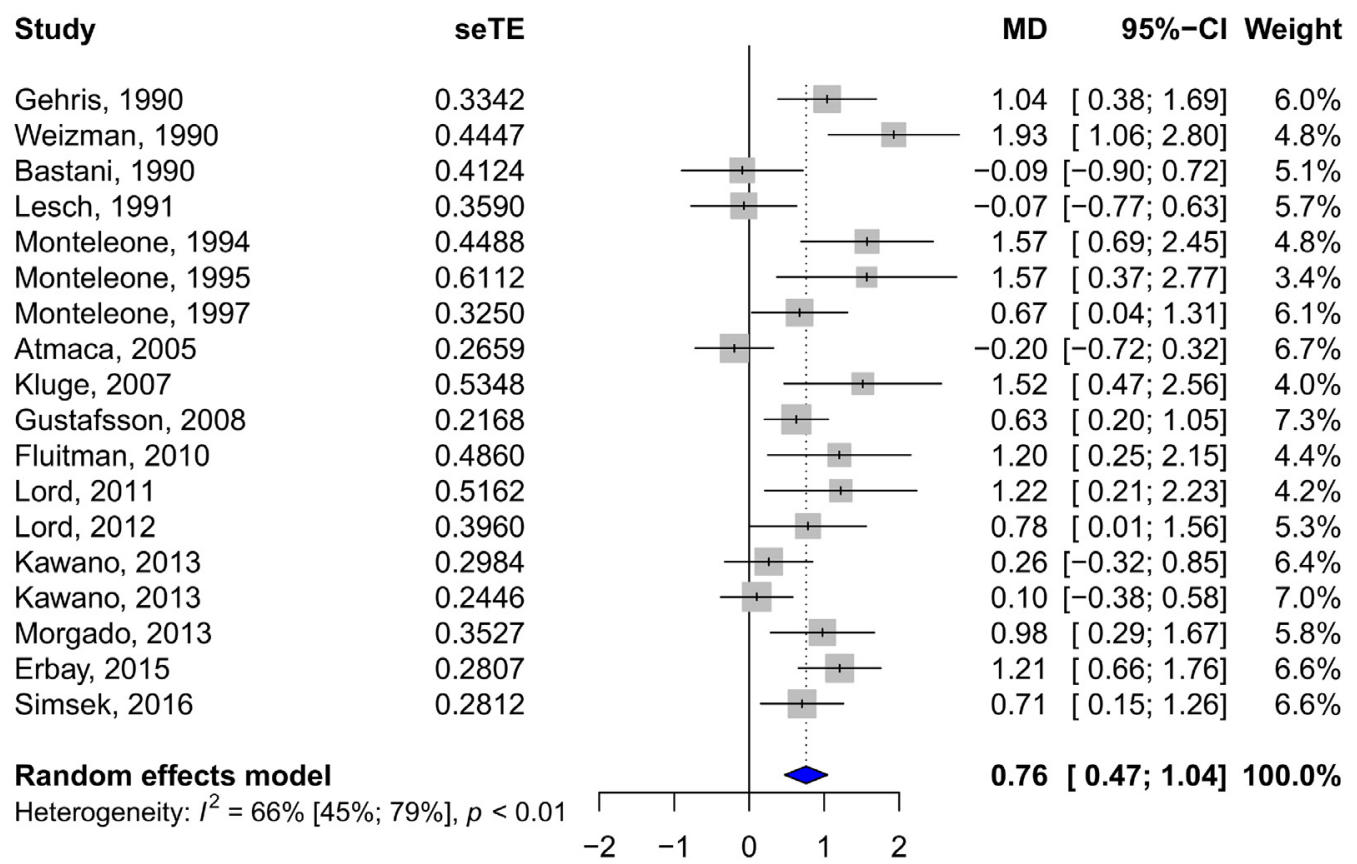

Fig. 2 Forest plot. Squares represents the effect sizes for individual studies. Horizontal lines correspond to the $95 \%$ confidence interval for the effect size. The solid vertical line represents the line of null effect; the dashed line represents the pooled effect size.
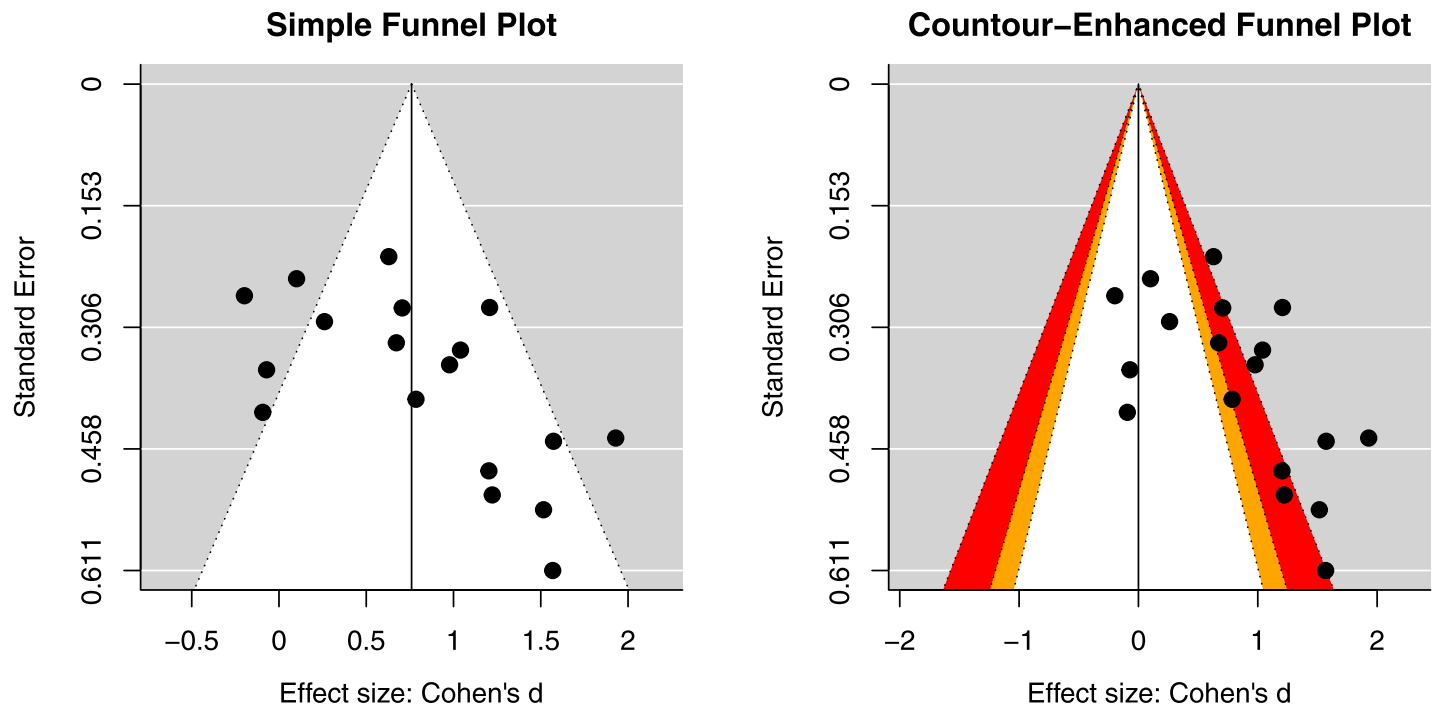

Fig. 3 Funnel plots. The $x$-axis represents the effect size; the $y$-axis represents the standard error of the estimate. On the left, the funnel plot is centered on the pooled effect size. On the right, the plot is centered at the null effect. Effects with statistical significance greater than $p=0.1$ are represented in the white area, between $p=0.05$ and $p=0.1$ in the orange area and between $p=0.01$ and $p=0.05$ in the red area. Black dots correspond to individual studies. (For interpretation of the references to color in this figure legend, the reader is referred to the web version of this article.)

\section{Discussion}

In this study, we conducted a systematic review and a meta-analysis to compare cortisol levels between patients with $O C D$ and healthy individuals. The meta-analytic results provided evidence for significantly enhanced cortisol levels in these patients.
The timing for cortisol collection seems to be a key factor in explaining different results from distinctive studies. In our meta-analysis, we found there is evidence for significant differences in cortisol levels between patients and healthy controls: the level of evidence for the afternoon period was found to be considerably weaker than that for the morning and entire day periods 
of collection, where this effect was substantially more pronounced.

Since the daily cortisol profile is known not to be significantly affected by acute changes in behavioral or environmental conditions (Oster et al., 2017), it is not likely that discrepancies in results from distinct studies are due to differences in the conditions under these were performed. However, it is important to note that while under normal circumstances cortisol levels reach its peak in the early morning and decline throughout the day, the same is not true in situations of chronic stress exposure, where this circadian rhythm is blunted (Oster et al., 2017). As such, it would be important to discard the possibility of having selected healthy individuals who might report elevated levels of perceived stress. In this same line of thinking, in an attempt to refine the conclusions of these studies, it would be key to consider the possible effects of disease severity and medication status (medicated vs non-medicated) in exacerbating or attenuating the stress response and, thus, observed cortisol levels. Additionally, factors such as disease subtype and age of onset, which are also known to contribute to disease heterogeneity, were not considered when establishing comparisons between groups.

It is also important to consider that a significant methodological heterogeneity among studies was observed, mostly at the level of cortisol assessment, where single versus average of assessments may significantly impact on the overall effect. In fact, comparison of the overall effects for each subgroup meta-analysis revealed a significant difference between coefficients, such that for studies using the average of multiple assessments the standardized coefficient was significantly higher when compared to studies focusing on single measurements.

Despite these limitations, the hypothesis of HPA axis dysregulation and abnormal circulating levels of cortisol in OCD patients seems likely. Previous animal studies have demonstrated the deleterious effects of chronic stress exposure, with stressed animals exhibiting cortico-striatal dysfunction and abnormalities in the orbitofrontal cortex, key neuronal circuits that have previously been shown to be involved in the pathophysiology of OCD (Dias-Ferreira et al., 2009; Fineberg et al., 2014; Torregrossa et al., 2008). Neuroimaging studies performed with human subjects point in the same direction (Anticevic et al., 2014; Hou et al., 2014; Soares et al., 2012; Stern et al., 2012).

It is important to note that an enhanced stress response and consequently elevated cortisol levels may also be related with the distress and increased anxiety that is known to be associated with OCD symptomatology (Cougle et al., 2011). In this line of thinking, in a study by Morgado et al. (2013), higher perceived stress as well as increased cortisol levels were found in OCD patients compared to healthy individuals. Interestingly, in OCD patients, a higher score of perceived stress (but not cortisol levels) was also shown to be positively correlated with disease severity as measured by Y-BOCS scale, and specifically at the level of the obsessive component (Morgado et al., 2013). This seems to be in line with the notion that obsessions are characterized by a higher anxiogenic and stressful quality, while compulsions constitute a means to relieve it (APA, 2013).
All these observations may point to the importance of evaluating the severity of obsessive and compulsive components in addition to global disease severity, since it is possible that it may impact on HPA axis activity and profile. The same might hold true for other factors such as anxiety, depressive symptoms or neuroticism since previous studies have found a positive relationship between higher trait anxiety and anxious arousal and elevated levels of cortisol during daytime (Doane et al., 2011; Harris et al., 2015; Polk et al., 2005; Portella et al., 2005). Additionally, in a recent study conducted with major depressive disorder (MDD) patients, OCD patients either with or without comorbid MDD and healthy subjects, the authors found no differences in HPA axis activity between diagnostic groups. However, when considering symptom dimensions such as trait anxiety, it was observed that OCD patients with higher trait anxiety demonstrated higher cortisol awakening response and decreased cortisol suppression after dexamethasone administration (Labad et al., 2018). Moreover, OCD patients with comorbid MDD presented with a flattened diurnal cortisol slope and lower levels of morning cortisol, which might be indicative of a differential pattern of HPA axis dysregulation in OCD or MDD alone compared to OCD with comorbid MDD (Labad et al., 2018). It is not yet clear why trait anxiety might have a distinct influence on HPA axis functioning in OCD patients, but different neurobiological mechanisms could explain differences found across distinct diagnostic groups (Labad et al., 2018).

In conclusion, HPA axis dysregulation, abnormal stress response and elevated cortisol levels may constitute an etiological factor in OCD pathology, but may also represent a consequence of the disease itself, or even both. In order to clarify this issue, further studies will be needed where limitations such as those mentioned above will be kept in mind.

\section{Role of the funding source}

The funder of the study had no role in study design, data collection, data analysis, data interpretation, or writing of the report. The corresponding author had full access to all the data in the study and had final responsibility for the decision to submit for publication.

\section{Contributors}

All authors designed the study. João Sousa-Lima and Pedro Silva Moreira performed selection of studies, initial screening and full-text analysis. Pedro Morgado, Nuno Sousa and Catarina Raposo-Lima were consulted for the selection of studies to be included in the analysis. Pedro Silva Moreira and João Sousa-Lima did the statistical analysis and were responsible for writing the first draft of the manuscript. Pedro Morgado and Nuno Sousa supervised all phases of the study. All authors wrote, revised and approved the final manuscript.

\section{Declaration of competing interest}

The authors declare no competing interests. 


\section{Acknowledgments}

This work was performed in the Life and Health Sciences Research Institute (ICVS), Minho University. Financial support was provided by grants from FCT - Foundation for Science and Technology fellowship grant (PhD-iHES program) to $\mathrm{Pe}$ dro Silva Moreira (reference PDE/BDE/113601/2015) and to Catarina Raposo-Lima (reference SFRH/BD/122959/2016), by Bial Foundation Research Project 206/16, by FEDER funds through the Operational Programme Competitiveness Factors - COMPETE and National Funds through FCT Foundation for Science and Technology under the project POCI-01-0145-FEDER-007038; and by the project NORTE-010145-FEDER-000013, supported by Norte Portugal Regional Operational Programme (NORTE 2020), under the PORTUGAL 2020 Partnership Agreement, through the European Regional Development Fund (ERDF).

\section{Supplementary materials}

Supplementary material associated with this article can be found, in the online version, at doi:10.1016/j.euroneuro. 2019.09.001.

\section{References}

Aguilera, G., 1994. Regulation of pituitary ACTH secretion during chronic stress. Front. Neuroendocrinol. 15, 321-350. doi:10. 1006/frne.1994.1013.

American Psychiatric Association, 2013. Diagnostic and Statistical Manual of Mental Disorders. In: Arlington, VA (Ed.), Diagnostic and Statistical Manual of Mental Disorders, fifth ed.. American Psychiatric Publishing.

Anticevic, A., Hu, S., Zhang, S., Savic, A., Billingslea, E., Wasylink, S., et al., 2014. Global resting-state functional magnetic resonance imaging analysis identifies frontal cortex, striatal, and cerebellar dysconnectivity in obsessive-compulsive disorder. Biol. Psychiatry 75 (8), 595-605. doi:10.1016/j.biopsych. 2013.10.021.

Atmaca, M., Tezcan, E., Kuloglu, M., Ustundag, B., 2005. Serum leptin levels in obsessive-compulsive disorder. Psychiatry Clin. Neurosci. 59 (2), 189-193.

Bastani, B., 1990. Prolactin and cortisol responses to MK-212, a serotonin agonist, in obsessive-compulsive disorder. Arch. Gen. Psychiatry 47 (9), 833.

Berridge, C., 2007. Noradrenergic modulation of arousal. Brain Res. 58, 1-17. doi:10.1016/j.brainresrev.2007.10.013.

Brander, G., Pérez-Vigil, A., Larsson, H., Mataix-Cols, D., 2016. Systematic review of environmental risk factors for obsessivecompulsive disorder: a propose roadmap from association to causation. Neurosci. Biobehav. Rev. 65, 36-62. doi:10.1016/j. neubiorev.2016.03.011.

Bremner, J.D., Krystal, J.H., Southwick, S.M., Charney, D.S., 1996. Noradrenergic mechanisms in stress and anxiety: II. Clinical studies. Synapse 23, 39-51.

Charmandari, E., Tsigos, C., Chrousos, G., 2005. Endocrinology of the stress response. Annu. Rev. Physiol. 67, 259-284. doi:10. 1146/annurev.physiol.67.040403.120816.

Cougle, J.R., Timpano, K.R., Fitch, K.E., Hawkins, K.A., 2011. Distress tolerance and obsessions: an integrative analysis. Depress Anxiety 28, 906-914.

Dias-Ferreira, E., Sousa, J.C., Melo, I., Morgado, P., Mesquita, A.R., Cerqueira, J.J., et al., 2009. Chronic stress causes frontostriatal reorganization and affects decision-making. Science 325 (5940), 621-625.

Doane, L.D., Franz, C.E., Prom-Wormley, E., Eaves, L.J., Mendoza, S.P., Hellhammer, D.H., et al., 2011. Negative emotionality, depressive symptoms and cortisol diurnal rhythms: analysis of a community sample of middle-aged males. Horm. Behav. 60, 202-209. http://dx.doi.org/10.1016/j.yhbeh.2011.05.003.

Erbay, L.G., Kartalci, S., 2015. Neurosteroid levels in patients with obsessive-compulsive disorder. Psychiatry Investig. 12 (4), 538.

Fineberg, N.A., Chamberlain, S.R., Goudriaan, A.E., Stein, D.J., Vanderschuren, L.J., Gillan, C.M., 2014. New developments in human neurocognition: clinical, genetic, and brain imaging correlates of impulsivity and compulsivity. CNS Spectr. 19 (1), 69-89.

Findley, D.B., Leckman, J.F., Katsovich, L., Lin, H., Zhang, H., Grantz, H., 2003. Development of the Yale Children's Global Stress Index (YCGSI) and its application in children and adolescents in Tourette's syndrome and obsessive-compulsive disorder. J. Am. Acad. Child Adolesc. Psychiatry 42, 450-457.

Fluitman, S.B.A.H.A., Denys, D.A.J.P., Heijnen, C.J., Westenberg, H.G.M., 2010. Disgust affects TNF- $\alpha$, IL-6 and noradrenalin levels in patients with obsessive-compulsive disorder. Psychoneuroendocrinology 35 (6), 906-911.

Gehris, T.L., Kathol, R.G., Black, D.W., Noyes, R., 1990. Urinary free cortisol levels in obsessive-compulsive disorder. Psychiatry Res. 32 (2), 151-158.

Gustafsson, P.E., Gustafsson, P.A., Ivarsson, T., Nelson, N., 2008. Diurnal cortisol levels and cortisol response in youths with obsessive-compulsive disorder. Neuropsychobiology 57 (1-2), 14-21.

Harris, A., Endresen Reme, S., Tangen, T., Hansen, A.M., Helene Garde, A., Eriksen, H.R., 2015. Diurnal cortisol rhythm: associated with anxiety and depression, or just an indication of lack of energy? Psychiatry Res. 228, 209-215. http://dx.doi.org/10. 1016/j.psychres.2015.04.006.

Herman, J.P., McKlveen, J.M., Solomon, M.B., Carvalho-Netto, E., Myers, B., 2012. Neural regulation of the stress response: glucocorticoid feedback mechanisms. Braz. J. Med. Biol. Res. 45, 292-298. doi:10.1590/S0100-879X2012007500041.

Higgins, J., Green, S., 2008. Cochrane Handbook for Systematic Reviews of Iterventions. Wiley-Blackwell.

Hou, J.M., Zhao, M., Zhang, W., Song, L.H., Wu, W.J., Wang, J., et al., 2014. Resting-state functional connectivity abnormalities in patients with obsessive-compulsive disorder and their healthy first-degree relatives. J. Psychiatry Neurosci. 39 (5), 304-311.

Jarcho, M.R., Slavich, G.M., Tylova-Stein, H., Wolkowitz, O.M., Burke, H.M., 2013. Dysregulated diurnal cortisol pattern is associated with glucocorticoid resistance in women with major depressive disorder. Biol. Psychol. 93 (1), 150-158.

Kawano, A., Tanaka, Y., Ishitobi, Y., Maruyama, Y., Ando, T., Inoue, A., et al., 2013. Salivary alpha-amylase and cortisol responsiveness following electrical stimulation stress in obsessive-compulsive disorder patients. Psychiatry Res. 209 (1), 85-90.

Kluge, M., Schüssler, P., Künzel, H.E., Dresler, M., Yassouridis, A., Steiger, A., 2007. Increased nocturnal secretion of ACTH and cortisol in obsessive compulsive disorder. J. Psychiatr. Res. 41 (11), 928-933.

Labad, J., Soria, V., Salvat-Pujol, N., Segalàs, C., Real, E., Urretavizcaya, M., et al., 2018. Hypothalamic-pituitary-adrenal axis activity in the comorbidity between obsessive-compulsive disorder and major depression. Psychoneuroendocrinology 93, 20-28. doi:10.1016/j.psyneuen.2018.04.008.

Lesch, K.P., Hoh, A., Disselkamp-Tietze, J., Wiesmann, M., Osterheider, M., Schulte, H.M., 1991. 5-Hydroxytryptamine1A receptor responsivity in obsessive-compulsive disorder. Comparison of patients and controls. Arch. Gen. Psychiatry 48 (6), 540-547.

Lord, C., Hall, G., Soares, C.N., Steiner, M., 2011. Physiological stress response in postpartum women with obsessive-compul- 
sive disorder: a pilot study. Psychoneuroendocrinology 36 (1), 133-138.

Lord, C., Steiner, M., Soares, C., Carew, C., Hall, G., 2012. Stress response in postpartum women with and without obsessive-compulsive symptoms: an fMRI study. J. Psychiatry Neurosci. 37 (2), 78-86.

Lucey, J.V., Butcher, G., Clare, A.W., Dinan, T.G., 1993. The anterior pituitary responds normally to protirelin in obsessive-compulsive disorder: evidence to support a neuroendocrine serotonergic deficit. Acta Psychiatr. Scand. 87 (6), 384-388.

McEwen, B., 1999. Stress and hippocampal plasticity. Annu. Rev. Neurosci. 22, 105-122. doi:10.1146/annurev.neuro.22.1.105.

Moher, D., Liberati, A., Tetzlaff, J., Altman, D.G.PRISMA Group, 2009. Preferred reporting items for systematic reviews and meta-analyses: the PRISMA statement. (Reprinted from annals of internal medicine). Phys. Ther. 89 (9), 873-880.

Monteleone, P., Catapano, F., Buono, G., Maj, M., 1994. Circadian rhythms of melatonin, cortisol and prolactin in patients with obsessive-compulsive disorder. Acta Psychiatr. Scand. 89 (6), 411-415.

Monteleone, P., Catapano, F., Tortorella, A., Di Martino, S., Maj, M., 1995. Plasma melatonin and cortisol circadian patterns in patients with obsessive-compulsive disorder before and after fluoxetine treatment. Psychoneuroendocrinology 20 (7), 763-770.

Monteleone, P., Catapano, F., Tortorella, A., Maj, M., 1997. Cortisol response to $\mathrm{d}$-fenfluramine in patients with obsessive-compulsive disorder and in healthy subjects: evidence for a gender-related effect. Neuropsychobiology 36 (1), 8-12.

Morgado, P., Freitas, D., Bessa, J.M., Sousa, N., Cerqueira, J.J., 2013. Perceived stress in obsessive-compulsive disorder is related with obsessive but not compulsive symptoms. Front. Psychiatry 4, 1-6 APR.

Morris, M.C., Compas, B.E., Garber, J., 2012. Relations among posttraumatic stress disorder, comorbid major depression, and HPA function: a systematic review and meta-analysis. Clin. Psychol. Rev. 32 (4), 301-315

Nasca, C., Bigio, B., Zelli, D., Nicoletti, F., McEwen, B., 2015. Mind the gap: glucocorticoids modulate hippocampal glutamate tone underlying individual differences in stress susceptibility. Mol. Psychiatry 20, 755-763. doi:10.1038/mp.2014.96.

Oster, H., Challet, E., Ott, V., Arvat, E., de Kloet, E.R., Dijk, D.J., Lightman, S., Vgontzas, A., Van Cauter, E., 2017. The functional and clinical significance of the 24-hour rhythm of circulating glucocorticoids. Endocr. Rev. 38 (1), 3-45. doi:10.1210/er. 2015-1080.

Phan, K., Fitzgerald, D., Nathan, P., Moore, G., Uhde, T., Tancer, M., 2005. Neural substrates for voluntary suppression of negative affect: a functional magnetic resonance imaging study. Biol. Psychiatry 57, 210-219. doi:10.1016/j.biopsych.2004.10.030.

Polk, D.E., Cohen, S., Doyle, W.J., Skoner, D.P., Kirschbaum, C., 2005. State and trait affect as predictors of salivary cortisol in healthy adults. Psychoneuroendocrinology 30, 261-272. http:// dx.doi.org/10.1016/j.psyneuen.2004.08.004.

Portella, M.J., Harmer, C.J., Flint, J., Cowen, P., Goodwin, G.M., 2005. Enhanced early morning salivary cortisol in neuroticism. Am. J. Psychiatry 162, 807-809. http://dx.doi.org/10.1176/ appi.ajp.162.4.807.

Reimold, M., Knobel, A., Rapp, M.A., Batra, A., Wiedemann, K., Ströhle, A., et al., 2011. Central serotonin transporter levels are associated with stress hormone response and anxiety. Psychopharmacology 213 (2-3), 563-572.

Ruscio, A.M., Stein, D.J., Chiu, W.T., Kessler, R.C., 2010. The epidemiology of obsessive-compulsive disorder in the National Comorbidity Survey Replication. Mol. Psychiatry 15, 53-63.

Sinha, R., 2008. Chronic stress, drug use, and vulnerability to addiction. Ann. N.Y. Acad. Sci. 1141, 105-130. doi:10.1196/annals. 1441.030.

Șimșek, Ș., Gençoğlan, S., Yksel, T., Kaplan, İ., Alaca, R., 2016. Cortisol and brain-derived neurotrophic factor levels prior to treatment in children with obsessive-compulsive disorder. J. Clin. Psychiatry 77 (7), e855-e859.

Soares, J.M., Sampaio, A., Ferreira, L.M., Santos, N.C., Marques, F., Palha, J.A., et al., 2012. Stress-induced changes in human decision-making are reversible. Transl. Psychiatry 2, e131.

Sousa, N., Almeida, O., 2012. Disconnection and reconnection: the morphological basis of (mal)adaption to stress. Trends Neurosci. 35, 742-751. doi:10.1016/j.tins.2012.08.006.

Stern, E.R., Fitzgerald, K.D., Welsh, R.C., Abelson, J.L., Taylor, S.F., 2012. Resting-state functional connectivity between fronto-parietal and default mode networks in obsessivecompulsive disorder. PLoS One 7 (5), e36356. doi:10.1371/ journal.pone.0036356.

Taylor, S., 2011. Etiology of obsessions and compulsions: a metaanalysis and narrative review of twin studies. Clin. Psychol. Rev. 31 (8), 1361-1372.

Toro, J., Cervera, M., Osejo, E., Salamero, M., 1992. Obsessivecompulsive disorder in childhood and adolescence: a clinical study. J. Child Psychol. Psychiatry 33, 1025-1037.

Torregrossa, M.M., Quinn, J.J., Taylor, J.C., 2008. Impulsivity, compulsivity, and habit: the role of orbitofrontal cortex revisited. Biol. Psychiatry 63 (3), 253-255.

Ursin, H., Ollf, M., 1993. Psychobiology of coping and defense strategies. Neuropsychobiology 7, 247-258.

Viechtbauer, W., 2010. Conducting meta-analyses in $\mathrm{R}$ with the metafor package. J. Stat. Softw. 36 (3), 1-48.

Walker, E., Diforio, D., 1997. Schizophrenia: a neural diathesis-stress model. Psychol. Rev. 104, 667-685.

Weizman, R., Gil-Ad, I., Hermesh, H., Munitz, H., Laron, Z., 1990. Immunoreactive beta-endorphin, cortisol, and growth hormone plasma levels in obsessive-compulsive disorder. J. Clin. Psychopharmacol. 13 (4), 297-302. 